\title{
Venusbeobachtungen 1917 am Passageninstrument der Sternwarte Babelsberg.
}

In der Mitteilung meiner Venusbeobachtungen I9 I5 (A. N. 202.377) habe ich erwähnt, daß das Pointieren der Ränder der Einstellung auf die Mitte der Venussscheibe. vorzuziehen sei. Diese Ansicht nachzuprüfen, war der Zweck der vorliegenden Reihe, die gelegentlich der oberen Konjunktion mit der Sonne ( 19 I 7 April 25) an demselben .Instrument und unter sonst denselben Bédingungen unternommen wurde.

Das Verhalten der Instrumentalfehler. war, bis auf eine kleine Unstimmigkeit Mitte April zwischen dem Azimut aus Polaris einerseits und den Miren andererseits, sehr zufriedenstellend. Ebenso erwiesen sich die beiden Riefleruhren $35^{6}$ und 346, die stündlich zur Kontrolle der Arbeitsuhr Tiede 3 hinzugezogen wurden, in ihrem Gang genug zuverlässig, um ihre aus den Abendwerten interpolierten Uhrkorrektionen den Tageswerten gleichstellen zu können (jede der beiden Riefleruhren mit dem Gewicht eines einzelnen Tagessterns). Zur Ableitung der Tageswerte $\Delta u_{T}$ dienten, neben den zodiakalen Fundamentalsternen, die ich im Anschluß an die Sonnenbeobachtungen des Fundamental-Katalogs beobachte, auch Zenitsterne aus Cassiopeia, Perseus und Auriga, beide Sterngruppen in ziemlich gleichem Anteil. Die scheinbaren Örter sind wieder dem B.J. entlehnt worden.

\begin{tabular}{|c|c|c|c|c|c|c|c|c|c|c|c|c|c|c|c|c|}
\hline \multicolumn{5}{|c|}{$\begin{array}{l}\text { Astronom. Datum } \\
\text { in Ortssternzeit }\end{array}$} & 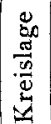 & $\begin{array}{c}\text { Aus } \\
\text { Tag. } \\
\text { beob. } \\
\Delta u_{T}\end{array}$ & 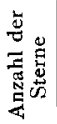 & $\begin{array}{c}\text { Aus } \\
\text { Abendw. } \\
\text { interpol. } \\
\Delta_{u} u_{A}\end{array}$ & $\underset{\Delta u}{\text { Angen. }}$ & $\begin{array}{l}\text { Verbess. } \\
\text { Uhrzeit } \\
\text { des } \\
\text { Durchg. }\end{array}$ & \multicolumn{3}{|c|}{$\alpha_{\mathrm{B}}$} & \multirow{2}{*}{$\frac{\alpha_{B}-\alpha_{R}}{+}$} & \multirow{2}{*}{$\frac{\left|\begin{array}{c}0 \\
0 \\
0 \\
0\end{array}\right|}{1 / 2}$} & Bemerkungen \\
\hline \multirow{4}{*}{$\begin{array}{c}\text { I9I } 7 \\
\text { März }\end{array}$} & 7 & $23^{h}$ & & ${ }^{n} 14: 7$ & $\mathrm{O}$ & $\stackrel{+}{+}$ & 2 & $\begin{array}{c}+ \\
\operatorname{II} \stackrel{\text { S. }}{2} 5^{.}\end{array}$ & $\stackrel{+}{\operatorname{II} 526}$ & $3^{580}:$ & \multicolumn{3}{|c|}{$6^{\mathrm{m}}+5^{\mathrm{s}} .06$} & & & \multirow{4}{*}{$\begin{array}{l}\text { \% sehr unruhig, aufgeblasen } \\
\text { dunstig }\end{array}$} \\
\hline & 23 & & 43 & $3 \cdot 7$ & $\mathrm{O}$ & I 2.79 & 6 & I 2.83 & I 2.80 & 51.28 & & 43 & 4.08 & 0.14. & I & \\
\hline & 24 & & 47 & 37.8 & $\mathrm{O}$ & .13 .10 & 5 & 13.10 & 13.10 & $25.05^{\circ}$ & & 47 & $3^{8.15}$ & O. I 4 & $\mathbf{I}$ & \\
\hline & 28 & o & 5 & $5 \mathrm{I.2}$ & $\mathrm{O}$ & $13.70^{\circ}$ & I & $13.7 \mathrm{I}$ & I $3.7 \mathrm{I}$ & $37.8 \mathrm{I}$. & 0 & 5 & $51.52^{\circ}$ & 0.09 & $\mathrm{I}$ & \\
\hline April & 3 & & 33 & 7.0 & $\mathrm{O}$ & I 4.18 & 7 & 14.23 & I 4.19 & 53.12. & & 33 & $7 \cdot 31$ & 0.04 & I & \\
\hline & 7 & & $5^{\mathrm{I}}$ & I 9.0 & W & I 4.76 . & 6 & $14.7 \mathrm{I}$ & I 4.75 & 4.25 & & $5 \mathrm{I}$ & 19.00 & 0.03. & $\mathbf{I}$ & o in Wolken \\
\hline & 10 & I & 5 & 0.3 & W & I 4.99 & 2 & I-4.98 & I $4.98^{\circ}$ & $45,43^{\circ}$ & I & 5 & 0.42 & 0.09 & $\mathbf{I}$ & schleierig \\
\hline & I 3 & & I 8 & 45.0 & W & I $5.25^{\circ}$ & 6 & I 5.24 & I 5.25 & 29.76 & & I 8 & 45.01 . & 0.00 & $1 / 2$ & instrumentelles Azimut aus Polaris und \\
\hline & I 4 & & 23 & 20.8 & W & I $5 \cdot 30^{\circ}$ & 2 & I 5.27 & I 5.29 & 5.63 & & 23 & $20.9 \mathrm{I}$. & 0.04 & $1 / 2$ & Miren nicht unbedeutend abweichend \\
\hline & 16 & & $3^{2}$ & $34 \cdot 3$ & W & I 5.45 & 5 & $5 \cdot 3^{6}$ & I $5.42^{\circ}$ & 18.89 & & 32 & $34 \cdot 3 \mathrm{I}$ & 0.02 . & $\mathbf{I}$ & dunstig, Bilder blähend \\
\hline Mai & 4 & 2 & 53 & $2 \cdot 5$ & $\mathrm{O}$ & I 6.86 & 4 & I 6.88 & I 6.86 . & 45.92 & 2 & 53 & 2.78 & 0.08 & $\mathbf{I}$ & \\
\hline & 6 & 3 & 2 & 48.8 & $\mathrm{O}$ & $16.3^{\circ}$ & 5 & I $6.39^{\circ}$ & r 6.33 & $32.7 I$ & 3 & 2 & 49.04 & $0.09^{\circ}$ & $\mathbf{I}$ & \\
\hline & 7 & & 7 & 43.6 & $\mathrm{O}$ & I $6 . \overline{1} 5$ & I 2 & - & I 6.15 & 27.68 & & 7 & 43.83. & 0.14 & $\mathbf{I}$ & von $1 u_{A}$ abgesehen, da genügende An- \\
\hline & ro & & 22 & $34 \cdot 7$ & $\mathrm{O}$ & I 5.43. & I 4 & - & I $5.43^{\circ}$ & I $9: 44$ & & 22 & 34.87 & 0.17 & $\mathbf{I}$ & zahl von Tagessternen \\
\hline & I 3 & & 37 & 34.1 & W & 14.94 & $\mathbf{I}$ & 15.03 & I 5.00 & 21.06 & & 37 & 36.06 & 0.14 & $\mathbf{I}$ & \\
\hline & 14 & & 42 & $3^{8.8}$ & $W$ & I $4.74^{\circ}$ & $\mathbf{I}$ & $\mathrm{I} 4.8 \mathrm{I}$. & I 4.79 & 23.99. & & 42 & $3^{8.78}$ & 0.16. & $\mathbf{I}$ & \\
\hline & 16 & & 52 & $47 \cdot 7$ & $W$ & I 4.28 . & 2 & 14.26. & $14.27^{\circ}$ & $33.29^{\circ}$ & & $5^{2}$ & $47 \cdot 57$ & 0.17 & $\mathbf{1}$ & schleierig \\
\hline & I 7 & & 57 & 53.6 & W & 13.99 & $\mathbf{I}$ & I 3.98 . & r 3.98 & 39.5 I : & & 57 & 53.50 & 0.03 & $1 / 2$ & hastig, Prisma nicht umgelegt \\
\hline & 20 & 4 & I 3 & 18.4 & W & $3.05^{\circ}$ & 5 & 13.05 & I $3.05^{\circ}$ & 5.29 & 4 & $x_{3}$ & I 8.34 & 0.12 & $\mathrm{r}$ & \\
\hline & $2 \mathrm{I}$ & & I 8 & 28.8 & W & I $2.79^{\circ}$ & 5 & I 2.78 & I $2.79^{\circ}$ & I 5.89 & & 18 & 28.68 & 0.10 & $\mathbf{I}$ & \\
\hline & 22 & & 23 & 40.2 & W & г 2.63 & 3 & I 2.67 & I 2.65 & 27.42 & & 23 & 40.07 & O.I I. & I & \\
\hline & 23 & & 28 & 52.5 & W & I $2.4^{6}$ & 4 & I 2.49 & I 2.47 & $39.93^{\circ}$ & & 28 & $52.40^{\circ}$ & 0.08 & $\mathbf{I}$ & \\
\hline & 24 & & 34 & 5.6 & $\mathrm{O}$ & I 2.20 & 4 & I 2.28 & I 2.23 & 53.59 & & 34 & 5.82 & 0.19 & $\mathbf{I}$ & \\
\hline Juni & 5 & 5 & 37 & 45.9 & $\mathrm{O}$ & 9.22 & 5 & $9.2 \mathrm{I}$ & 9.22 & 36.80 & 5 & 37 & 46.02 & $0 . \mathrm{II}^{\circ}$ & $\mathbf{I}$ & \\
\hline & 6 & & 43 & 8.0 & $\mathrm{O}$ & $8.95^{\circ}$ & 4 & 8.98 & $8.96^{\circ}$ & $59.06^{\circ}$ & & & 8.03 & 0.12 & $\mathbf{I}$ & \\
\hline & 8 & & 53 & 52.9 & 0 & $8.46^{\circ}$ & 3 & $8.51 \cdot 1$ & 8.48 & $44.5^{\circ}$ & & 53 & 52.98 & 0.14. & I & \\
\hline
\end{tabular}

In der Annahme, daß die Werte B - R bloß $z$ ufällige Fehler- enthalten, bekommt man die folgenden Normalwerte für die Ephemeridenkorrektion $\boldsymbol{L} \alpha$ :

$$
\begin{aligned}
& \text { I } 9 \text { I } 5 \text { Sept. I } 7 \text { +o. } 137 \pm 0.012 \\
& \text { I91 } 7 \mathrm{Mai} 3 \text { to.1 } 12 \pm 0.010
\end{aligned}
$$

Der mittl. Fehler einer Venusbeobachtung, wenn man vom deutlich ausgesprochenen systematischen Unterschied der $\mathrm{B}-\mathrm{R}$ vor und nach der Konjunktion ("jährliche Refraktion «) absieht, ergibt für beide Reihen \pm 0.55 . Will man aber diesen systematischen Unterschied in Rechnung ziehen, so sinkt der m. F. einer Venusbeobachtung für die vorliegende Reihe (mit Ausschluß der ersten Beobachtung vom I 5 . März) auf \pm 0.04 und für die alte auf \pm 0.045 .

Wenn auch in diesem Ergebnis die Überlegenheit des Ränder-Pointierens nicht genug $z u$ Tage tritt, so ist dieses Verfahren dem Pointieren der Mitte des Planeten schon aus dem Grunde vorzuziehen, daß das letztere wegen einer systematischen Änderung der Auffassung der visuellen Mitte unbedingt größere Gefahr in sich birgt.

Sternwarte Babelsberg, I 9 I 8 Juni.

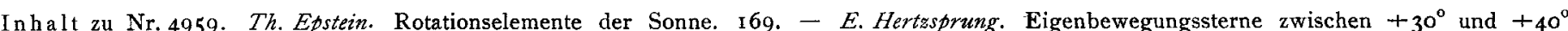
Deklination. I7I. $-F$. Kepinski. Venusbeobachtungen I9I7 am Passageninstrument der Sternwarte Babelsberg. I75. 